\title{
Immunoglobulin gene repertoire diversification and selection in the stomach - from gastritis to gastric lymphomas
}

\section{Miri Michaeli ${ }^{1+}$, Hilla Tabibian-Keissar ${ }^{1,2 \dagger}$, Ginette Schiby ${ }^{2 \dagger}{ }^{+}$, Gitit Shahaf $^{1}$, Yishai Pickman ${ }^{1}$, Lena Hazanov ${ }^{1}$, Kinneret Rosenblatt ${ }^{2}$, Deborah K. Dunn-Walters ${ }^{3}$, Iris Barshack ${ }^{2,4 \neq}$ and Ramit Mehr ${ }^{1 * \neq}$}

${ }^{1}$ The Mina and Everard Goodman Faculty of Life Sciences, Bar-llan University, Ramat Gan, Israel

2 Department of Pathology, Sheba Medical Center, Ramat Gan, Israel

${ }^{3}$ Division of Immunology, Infection, and Inflammatory Diseases, King's College London School of Medicine, London, UK

${ }^{4}$ Sackler Faculty of Medicine, Tel Aviv University, Tel Aviv, Israel

Edited by:

Michal Or-Guil, Humboldt University

Berlin, Germany

\section{Reviewed by:}

Jose Faro, Universidade de Vigo,

Spain

Andrew M. Collins, University of New

South Wales, Australia

*Correspondence:

Ramit Mehr, The Mina and Everard Goodman Faculty of Life Sciences,

Bar-Ilan University, Ramat Gan 52900, Israel

e-mail: ramit.mehr@biu.ac.il

${ }^{\dagger}$ Miri Michaeli, Hilla Tabibian-Keissar and Ginette Schiby have contributed equally to this work.

* rris Barshack and Ramit Mehr have equally supervised this study.
Chronic gastritis is characterized by gastric mucosal inflammation due to autoimmune responses or infection, frequently with Helicobacter pylori. Gastritis with $H$. pylori background can cause gastric mucosa-associated lymphoid tissue lymphoma (MALT-L), which sometimes further transforms into diffuse large B-cell lymphoma (DLBCL). However, gastric DLBCL can also be initiated de novo. The mechanisms underlying transformation into DLBCL are not completely understood. We analyzed immunoglobulin repertoires and clonal trees to investigate whether and how immunoglobulin gene repertoires, clonal diversification, and selection in gastritis, gastric MALT-L, and DLBCL differ from each other and from normal responses. The two gastritis types (positive or negative for $\mathrm{H}$. pylori) had similarly diverse repertoires. MALT-L dominant clones (defined as the largest clones in each sample) presented higher diversification and longer mutational histories compared with all other conditions. DLBCL dominant clones displayed lower clonal diversification, suggesting the transforming events are triggered by similar responses in different patients. These results are surprising, as we expected to find similarities between the dominant clones of gastritis and MALT-L and between those of MALT-L and DLBCL.

Keywords: B-cells, gastritis, H. pylori, MALT lymphoma, DLBCL, Ig gene, repertoire, somatic hypermutation

\section{INTRODUCTION}

Chronic gastritis is a common disorder characterized by chronic inflammation of gastric mucosa. In acute gastritis, patients suffer from dyspeptic symptoms including epigastric burning, distention or bloating, belching, episodic nausea, flatulence, and halitosis. In contrast, most patients with chronic gastritis are asymptomatic (1). One of the major causes of gastritis is bacterial infection, most frequently with Helicobacter pylori (H. pylori). H. pylori are Gram-negative bacteria that are present in the gastric mucosa of more than $50 \%$ of people and may persist lifelong unless treated (2). H. pylori are resistant to elimination by the immune response so the immune system fails to remove the infection effectively (3). Previous studies have shown a strong association between gastritis and $H$. pylori infection, at least in the early stages of gastritis $(3,4)$. Although rare, organisms other than H. pylori (e.g., Mycobacterium avium-intracellulare, Herpes simplex, Cytomegalovirus, and Epstein-Barr virus) can invade the

Abbreviations: AML, acute myeloid leukemia; B-CLL, chronic lymphocytic leukemia; BCR, B-cell receptor; CDR, complementary determining region; $\mathrm{CI}$, confidence intervals; CLN, control lymph node; DLBCL, diffuse large B-cell lymphoma; FL, follicular lymphoma; GHP, gastritis with $H$. pylori background; GNHP, gastritis without $H$. pylori background; $H$. pylori, Helicobacter pylori; HTS, high-throughput sequencing; Indels, insertions and/or deletions; MALT, mucosa-associated lymphoid tissue; MALT-L, MALT lymphoma; MID, molecular identification; MM, multiple myeloma; SHM, somatic hypermutation. gastric mucosa and cause inflammation $(5,6)$. Gastritis can also be initiated de novo, as an autoimmune disease (7). In either case, prolonged antigenic stimulation causing chronic inflammation might further contribute to the development of some malignancies (8), such as gastric mucosa-associated lymphoid tissue (MALT) lymphoma (9-16).

Mucosa-associated lymphoid tissue lymphoma (MALT-L) is a low-grade B-cell lymphoma. It grows slowly and remains confined to one organ for a relatively long time. Stomach MALT-L exemplifies the close link between chronic inflammation and lymphomagenesis. B-cells of MALT-L are related to normal marginal zone cells. Their IgH variable region gene sequences exhibit features of post germinal center B-cells, such as somatic hypermutation (SHM), implying that the clone has expanded in the presence of an antigen (17). MALT-L is often associated with bacterial infection, most commonly by $H$. pylori bacterium $(7-9,15-17)$.

A possible outcome of low-grade B-cell lymphomas such as MALT-L is the transformation into a more aggressive lymphoma such as diffuse large B-cell lymphoma (DLBCL) $(18,19)$. Gastric DLBCL is a fast-growing, aggressive B-cell malignancy characterized by diffuse proliferation of large neoplastic lymphoid B-cells $(20,21)$. DLBCL is known to represent a heterogeneous group of malignancies, comprising either germinal center-like cells exhibiting intra-clonal diversity or "activated B-cell-like" cells, which do not $(22,23)$. 
During the clonal expansion of B-cells in response to an antigen, Ig gene sequences from clonally related B-cells (i.e., B-cells that are derivatives of the same B-cell ancestor) accumulate mutations via SHM and thus diversify. Clonally related cells are identified by identical V(D)J segments and by highly homologous sequences of the complementary determining region (CDR) 3 of their Ig genes. An easy way to track and analyze the relationships between clonally related Ig gene sequences is by using lineage trees. The tree root is the ancestor sequence, usually the rearranged, pre-mutation sequence. Each tree node represents a single mutation (point mutation, insertion, or deletion). Lineage trees have been used in order to quantify the differences between the dynamics of SHM and antigen-driven selection in different lymphoid tissues, species, and disease situations. Our lineage trees-based mutation analysis has demonstrated its usefulness in previous studies of aging (24), autoimmunity (25-28), and chronic inflammation (29). Recent work on B-cell malignancies done in our lab (30-32) showed differences in tree properties between lymphomas and controls. Lymphoma trees were more branched and had longer trunks compared to controls, indicating a higher intraclonal diversification and a longer mutational history. Intra-clonal diversification was also shown in chronic lymphocytic leukemia cases (33-35), in marginal zone lymphoma cases $(36,37)$ and in intestinal DLBCL cases (21). In addition, lymphoma and controls exhibited similar mutation rates and same SHM motifs. Follicular lymphoma (FL), which is considered a less aggressive lymphoma, displayed higher diversity than DLBCL and highest recent diversification events, suggesting that the more aggressive lymphoma diversifies the least (38-40).

In the present study, we used repertoire, lineage tree, and mutation analyses to investigate whether and how B-cell repertoires, clonal diversification, and selection mechanisms in gastritis, gastric MALT-L, and DLBCL differ from each other and from normal responses. The two types of gastritis (positive or negative for $H$. pylori) were found to have similar repertoires and diversification. MALT-L clones were found to be more diversified and had longer mutational histories compared with all other conditions, but the dominant clones of MALT-L (defined as the largest clones in each sample) were different from those of all other conditions. DLBCL dominant clones, however, displayed lower diversification. These results are surprising, as we expected to find similarities between the dominant clones of gastritis and MALT-L and between those of MALT-L and DLBCL, according to the hypothesis that these are often sequential steps of inflammation and transformation.

\section{RESULTS \\ REPERTOIRES IN GASTRITIS WITH H. PYLORI BACKGROUND WERE AS DIVERSE AS THOSE IN GASTRITIS NEGATIVE FOR H. PYLORI, AND CONTAINED SIMILAR V(D)J COMBINATIONS}

We compared the repertoires in both types of gastritis, with H. pylori background (GHP) or without $H$. pylori background (GNHP), and examined the differences between them. We expected the repertoire in GHP to be less diverse due to the response to the bacterium, as previous studies showed that monoclonality is frequently found in GHP samples [(41-43) and others]. In contrast to our expectation, the confidence intervals (CI) of alpha, beta, and gamma diversity indices of both orders were

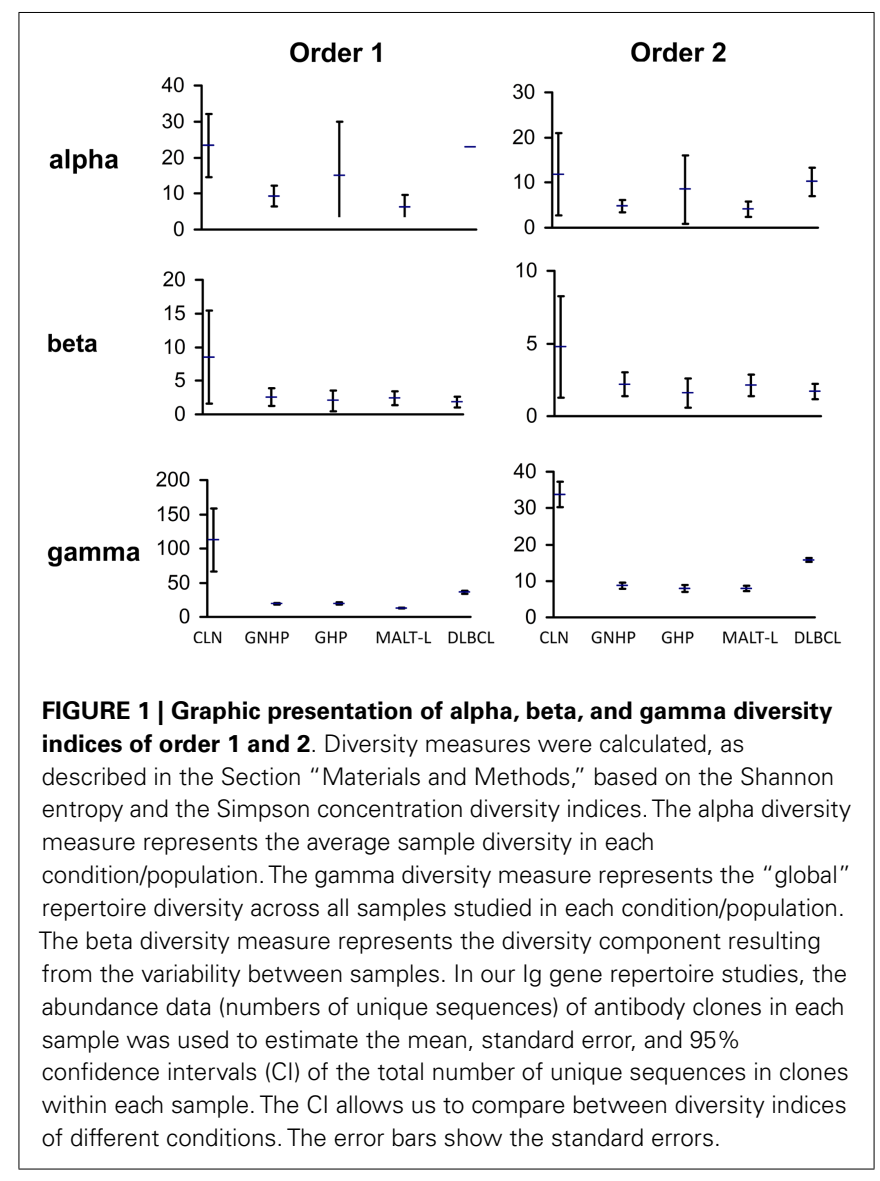

overlapping (Figure 1), implying the average individual biopsy diversities, the variability of diversities between individual biopsies, and the overall pool diversities in GHP and GNHP were not statistically different. Indeed, most V(D)J combinations observed were expressed in both gastritis types (Figures 2A,B).

Gastritis with $H$. pylori background and GNHP were the most similar conditions (similarity index of 0.543 , Table 1), although one GHP sample (the second GHP sample in Table S1 in Supplementary Material) had an extremely high alpha diversity index compared to the other two samples (data not shown). This contradicts our expectation of narrower repertoires in GHP samples due to the presence of $H$. pylori. However, if the one highly diverse GHP sample is excluded from the analysis, the confidence interval of alpha of GHP becomes narrower (3.9-11.25), and lower than that of GNHP. It is possible that the highly diverse sample reflected additional ongoing responses.

VH1-3/JH4 was a common combination in both GNHP and GHP VH-JH repertoires, but not so prominent in repertoires of other conditions (Figures 2A,B). These combinations contained several DH genes in both GNHP and GHP. However, identification of $\mathrm{D}$ genes should be taken with caution, as SoDA always finds a D gene, even when this is based on too-few nucleotides to be reliable. Table 2 summarizes all common combinations and genes found in our study and their relationships with other clinical conditions as implicated in the literature. 


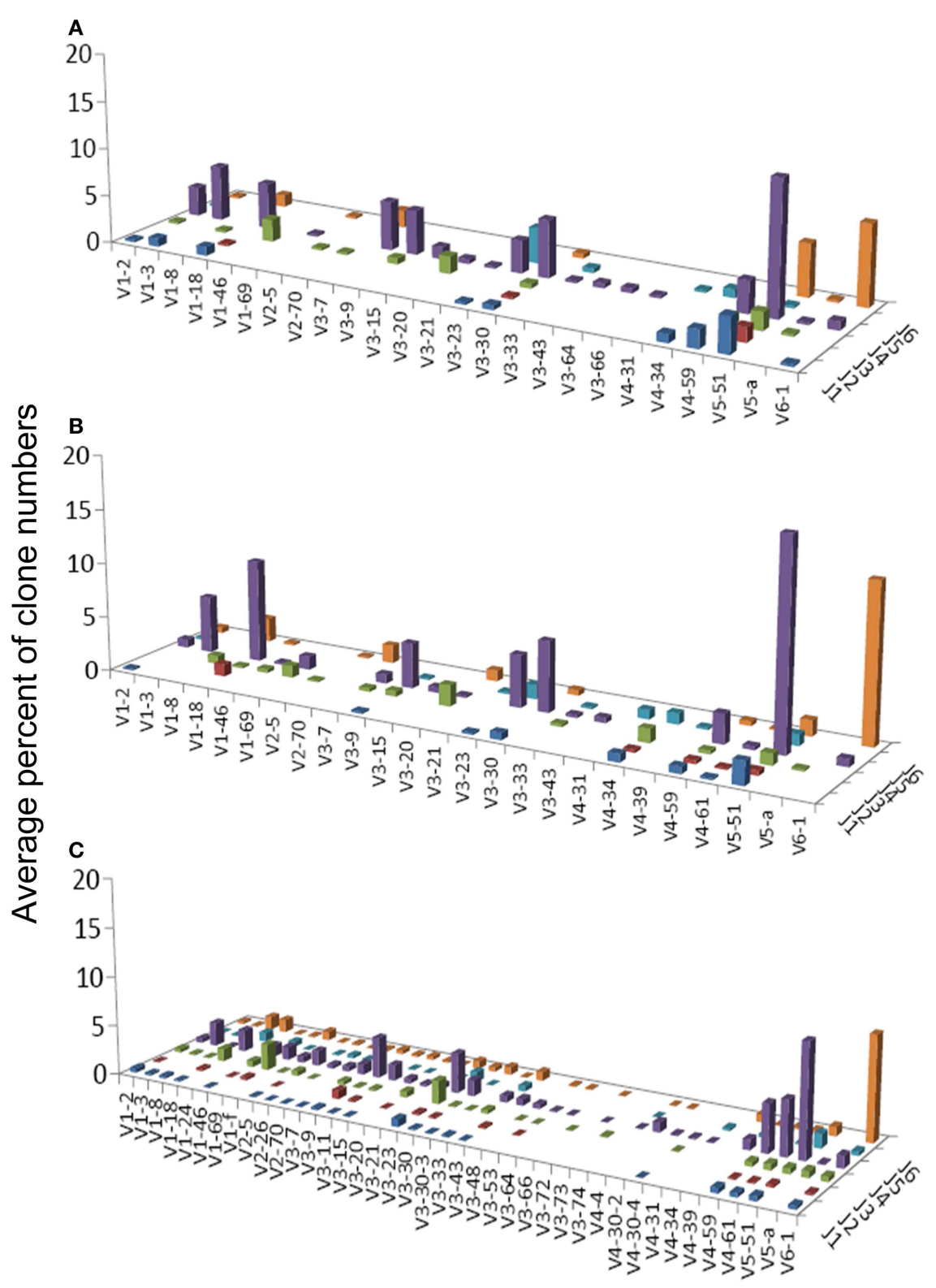

FIGURE 2 | Average percentages of clones in each VH-JH combination, in (A) GHP, (B) GNHP, and (C) CLN samples.

Table 1 | The average similarity between each pair of conditions

\begin{tabular}{lccc}
\hline & CLN & GNHP & GHP \\
\hline CLN & 0.211 & 0.295 & 0.343 \\
GNHP & & 0.408 & 0.543 \\
GHP & & & 0.478
\end{tabular}

Similarity measures were calculated between all clones in all samples in the compared conditions. In lymphomas, only the dominant clone(s) are relevant, as the rest of the clones in each sample represent other B-cells present in the tissue, which are not related to the malignancy. Thus, MALT-L and DLBCL are not included in the calculation of similarity measures because the dominant clones in these conditions cannot be compared to the full repertoire samples from other conditions.
In the case of GNHP, the DH3 and DH6 families were found to be preferred, as combinations of V6D3J6 and V6D6J6 were used significantly more than expected (Table 3; Figure 2B). Other overexpressed DH genes were used in less prominent combinations in the observed repertoire. Other combinations used in GNHP and GHP, such as VH3-7, VH3-23, and VH3-30 - all with JH4 - used several $\mathrm{DH}$ genes from different $\mathrm{DH}$ families.

Gastritis without H. pylori background and GHP presented almost identical gene usage patterns, having VH5$51 / \mathrm{JH} 4$ and VH6-1/JH6 as the two most frequent combinations (Figures 2A,B). VH5-51 and VH6-1 have been shown to often participate in earlier stages of repertoire development via positive selection by auto-antigens (Table 2). These two combinations also appeared in our control lymph node (CLN) samples (Figure 2C), 
Table 2 | A summary of frequent combinations and genes in conditions from our study and from other studies.

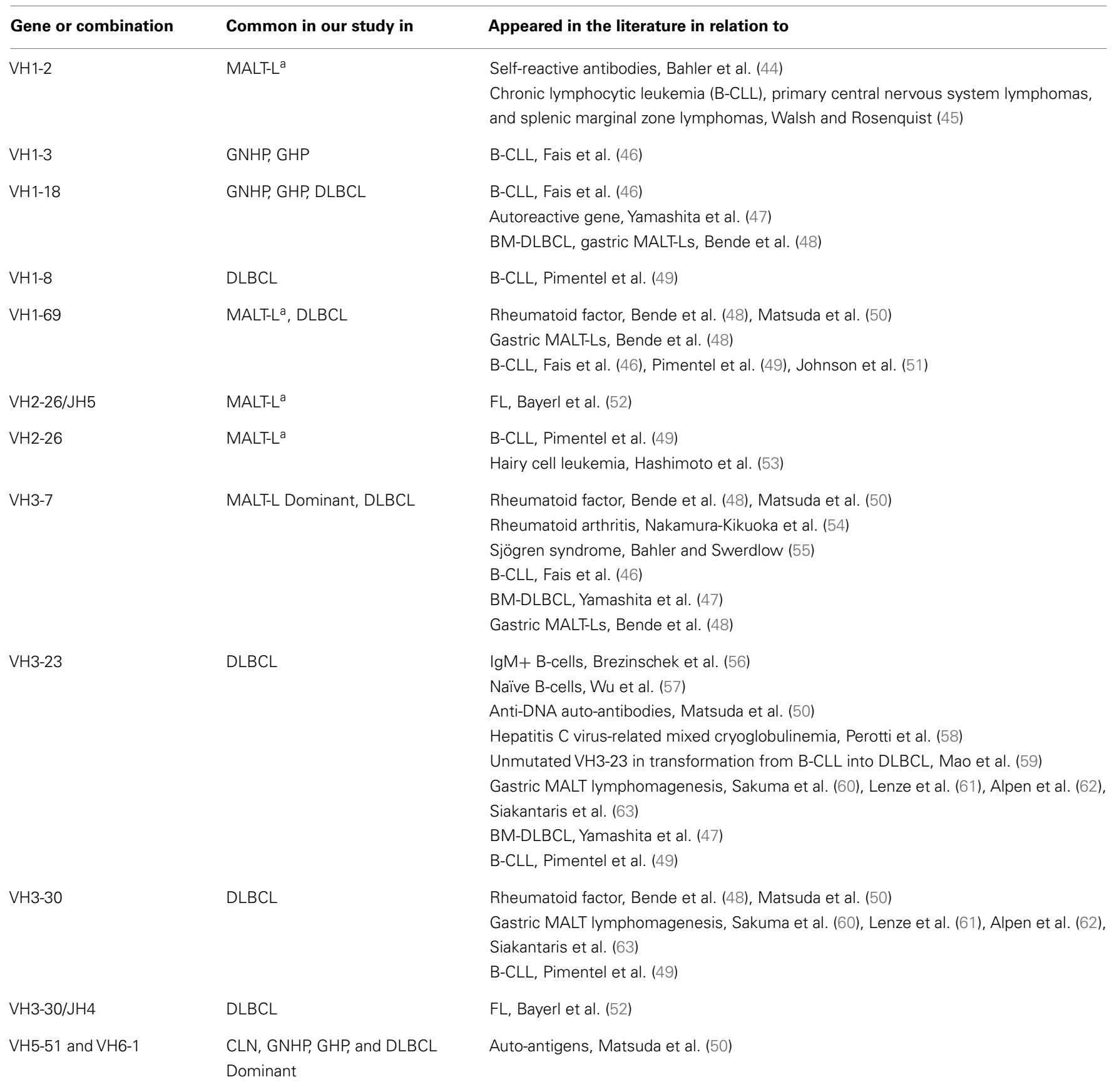

The dominant segments are marked with "Dominant" indication. The dominant segments/combinations are those that appeared in the largest clone in each sample. ${ }^{a}$ Represents frequent genes or combinations in the repertoire of unique sequences.

so they probably have no specific connection to gastritis or $H$. pylori response. However, the VH1-18/JH4 combination was more frequently used in GNHP than in GHP, and was not prominently observed in other conditions. As VH1-18 is an autoreactive gene and was found in several gastric MALT-Ls (Table 2), VH1-18 may be involved in the development of gastritis regardless of the presence of $H$. pylori.
One combination was over-expressed in both types of gastritis (V3D0J5), and two combinations (V6D3J6 and V6D6J6, Table 3) were over-expressed in GNHP (as dominant clones) and in DLBCL samples (not the dominant clones). As can be seen in Figures 2B,C and 3A, many sequences used the V6-J6 combination in GNHP and DLBCL, but also in the controls. Thus, this combination is very frequent in immune responses, and cannot be ascribed to a 
Table 3 |VDJ combinations that were over-expressed in each condition $^{\mathrm{a}}$.

\begin{tabular}{llcc}
\hline Condition & Combination & p-Value & Mean difference $^{\text {b }}$ \\
\hline GNHP & V2D1J6 & 0.009 & 3.57 \\
& V3D5J4 & 0.002 & 4.38 \\
& V3D0J5 & 0.001 & 7.84 \\
& V6D6J6 & 0.035 & 3.84 \\
& V6D3J6 & 0.042 & 3.60 \\
GHP & V3D0J5 & 0.035 & 10.44 \\
& V5D3J2 & 0.015 & 3.26 \\
DLBCL & V1D1J6 & 0.011 & 1.30 \\
& Dominant & & \\
& V1D4J3 & 0.000 & 4.94 \\
& V4D6J4 & 0.046 & 2.70 \\
& V5D1J4 & 0.001 & 1.80 \\
& Dominant & \\
& V5D7J4 & & 2.20 \\
& V6D6J6 & 0.036 & 3.47 \\
& V6D3J6 & 0.000 & 3.50
\end{tabular}

aThere were no VDJ combinations that where over-expressed in MALT-L samples, thus MALT-L does not appear in the table.

${ }^{b}$ Represents the value of $\log _{2}$ (observed/expected).

'This combination was found in DLBCL samples number 1, 2, 3 (sample numbers according to Table $S 1$ in Supplementary Material). The dominant combinations are those that appeared in the largest clone in each sample.

'This combination was found in DLBCL sample number 5 .

specific condition. However, the combination V3D0J5 may represent an antibody that is effective in the gastric environment, related to inflammatory processes, or participates in both.

To conclude, both types of gastritis presented similar repertoires and diversity properties, in contrast to our expectation.

\section{GASTRIC MALT-L EXHIBITED UNIOUE V(D)J COMBINATIONS}

Several studies have demonstrated that gastric MALT-L is often associated with a bacterial infection, most commonly by H. pylori; another association has been revealed between gastritis and gastric MALT-L (11-13). Therefore, we expected to find similar $\mathrm{V}(\mathrm{D}) \mathrm{J}$ combinations when comparing the two conditions. Surprisingly, the dominant MALT-L V(D)J combinations were very different from those in GHP. While GHP showed an extensive use of JH4 family genes and several common combinations, of which the most frequent was VH5-51/JH4, in MALT-L dominant clones were VH3-7/JH4, VH1-69/JH6, and VH1-2/JH1. VH3-7 is frequently found in rheumatoid factors and was selectively expressed by patients with rheumatoid arthritis and Sjögren syndrome. Preferential use of these genes and combinations has been reported in several types of lymphomas and leukemias (Table 2).

Table S2 in Supplementary Material presents the combinations that were over-expressed in one condition while underrepresented in the other. It can be seen (from the "Mean deviation" column) that over-expressed combinations were almost absolutely from either MALT-L or DLBCL samples (dominant clones only). As DLBCL contained dominant combinations that also appeared in other conditions, this supports the observation of different dominant combinations in MALT-L compared to those observed in other conditions. These combinations may relate to the malignancy, but this remains to be explored.

We also compared the dominant clones in DLBCL and MALT$\mathrm{L}$ samples, as in some cases DLBCL appears in association with MALT-L $(18,19)$. DLBCL is considered in these cases to result from clonal transformation of large cells within the low-grade lymphoma $(64,65)$. Hence, we expected to identify similar segment combinations on the dominant clones from the two conditions. However, the dominant clones of MALT-L samples were different from those of DLBCL (Figures 3A,B). As mentioned above, the dominant clones in MALT-Ls were VH3-7/JH4, VH169/JH6, and VH1-2/JH1, while in DLBCLs these combinations were found, but were not the dominant clones. VH5-51/JH4 and VH6-1/JH6 were frequent combinations in all conditions, except in MALT-L, suggesting they may have some advantage in binding common antigens. In terms of unique sequences, MALT-Ls presented completely different dominant combinations from DLBCL (VH1-2/JH1, VH1-69/JH6, VH2-26/JH5, and VH3-7/JH4, data not shown). Preferential use of these genes and combinations has been reported in several types of lymphomas and leukemias (Table 2). In addition, VH3-7/JH4, which was the most frequent combination in MALT-L dominant clones, appeared in all other conditions but with dramatically lower numbers. As mentioned above, VH3-7 participates in the formation of autoantibodies and was found in several gastric MALT-Ls. Some MALT-Ls were found to use VH genes previously associated with auto-reactivity. This suggests that B-cells in MALT-L react with self-antigens (66), different from those that arouse in GHP and DLBCL responses.

\section{MALT-L DOMINANT CLONES HAD LONGER DIVERSIFICATION HISTORY, IN CONTRAST TO DLBCL CLONES}

Lineage trees of the MALT-L dominant clones had significantly longer trunks $(\mathrm{T})$ and path lengths (PLmin), which are tree length measures, than all other conditions (Figure 4; Figure S1 in Supplementary Material). In addition, according to the correlation of tree properties with the dynamic parameters of the secondary B-cell response (67), longer trunks correlate with a lower initial affinity, and longer paths also correlate with a lower selection threshold. This suggests that diversification history in MALT-L dominant clones was longer than that of other conditions.

On the contrary, DLBCL dominant clones had significantly shorter trunks and path lengths than those of GHP (and MALT$\mathrm{L}$ ), and in general, the lowest tree length measures. Dominant clones of DLBCL presented similar tree length measures to those of CLN (Figure 4; Figure S1 in Supplementary Material). This is in line with the above-described observation of similarity between DLBCL and CLN. The shorter lengths observed in DLBCL, which correlate with high initial affinity and selection threshold, may indicate a shorter diversification process compared to MALT-L and GHP (21). A possible explanation for this is that, because MALT-L 


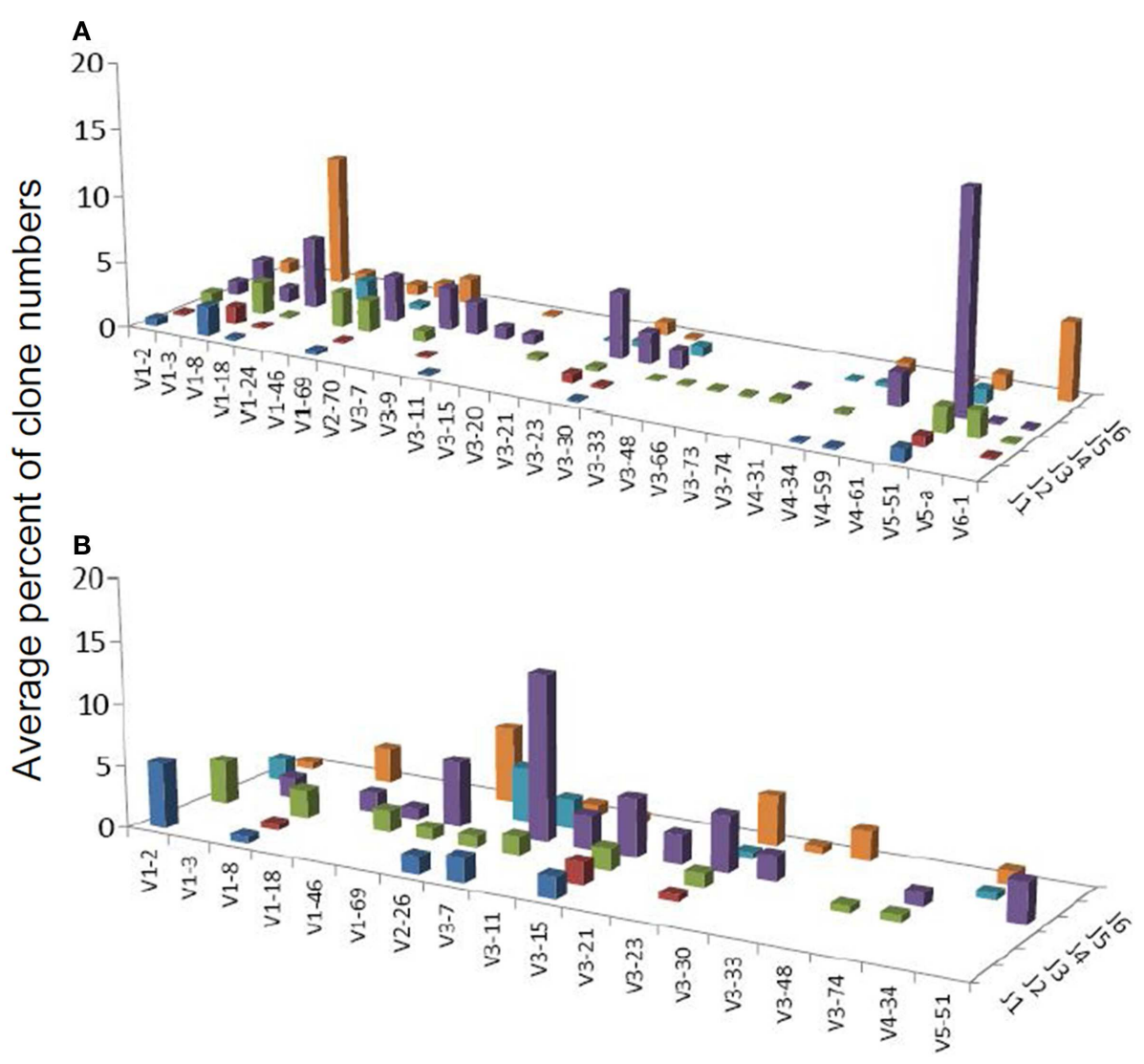

FIGURE 3 | Average percentages of clones in each VH-JH combination, in (A) DLBCL and (B) MALT-L samples.

is an indolent lymphoma and DLBCL is an aggressive lymphoma, the latter usually has less time to diversify until it is discovered and treated. Figures S2 and S3 in Supplementary Material show representative examples for MALT-L and DLBCL dominant trees, respectively.

The fact that MALT-L dominant clones had larger trunks, path lengths, and distance from the root to any split node, thus probably lower initial affinity than DLBCL dominant clones, suggests that DLBCL dominant clones started as responses to specific (yetunknown) antigens, with probably higher initial affinity than the responses that initiated MALT-Ls. That is, high affinity and vigorous response may be risk factors for aggressive lymphoma development. In terms of selection, these results show that selection thresholds in MALT-L dominant clones were the lowest among all other conditions. Low selection pressure may simply be the result of abundance of antigen, and this may indeed be the case in gastric MALT-Ls.

Dominant clones from the two types of gastritis presented similar tree length measures, which correlate with the observed similar repertoires.

\section{DISCUSSION}

In this study, we investigated the relationships between four related conditions of the stomach: gastritis positive or negative for H. pylori, gastric MALT-L, and gastric DLBCL. As previous studies showed, these conditions sometimes appear successively, as prolonged stimulation during chronic gastritis may result in the development of gastric MALT-L, which in some cases further transforms into DLBCL. We examined the clonal repertoires of the $\mathrm{IgH}$ variable region genes (or in the case of lymphomas, the dominant clones, which are defined as the largest clone in each sample) and the lineage tree characteristics in each condition, in order to find similarities or differences between these conditions.

Both types of gastritis presented similar IgH variable region gene repertoires and lineage tree characteristics, in contrast to our pre-study assumptions. However, although the GNHP biopsies were negative for $\mathrm{H}$. pylori, it could be present in the tissue in undetectable amounts, and thus affect the repertoire of the Bcells in its surroundings. Moreover, both types of gastritis used the VH1-18 gene, which may be involved in the development of gastritis regardless of the presence of $H$. pylori; this remains to be elucidated, as this was not the dominant combination in both type of gastritis. We expected the repertoire in GHP to be less diverse than that of GNHP due to the response to the bacterium, which is expected to elicit only specific clones. In contrast to our expectations, GHP samples showed at least as diverse repertoires as GNHP (Figures $\mathbf{1}$ and $\mathbf{2 A}, \mathbf{B}$ ). An explanation for a high diversity in GHP might be the phase variation of $H$. pylori, which is the generation of intra-strain diversity that is important for bacterial 


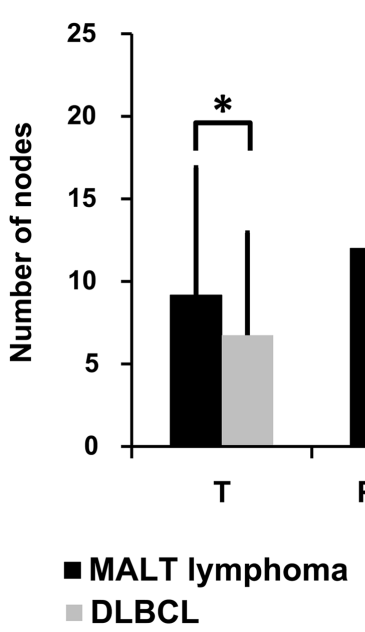

FIGURE 4 | Lineage tree analysis - comparison between dominant clones from the three MALT-L samples (24 trees) and the five DLBCL samples (47 trees). There was more than one tree per sample, as we included all clones with the same $\mathrm{VH}$ and $\mathrm{JH}$ genes (but different alleles) in the dominant clone in each sample, because they might be related to the dominant clone and falsely attributed to other alleles. Significant differences were found in trunk length $(T)$ and minimal path length (PLmin). An asterisk $\left({ }^{*}\right)$ represents $p$-value $<0.01$; two asterisks $\left({ }^{* *}\right)$ represents $p$-value $<0.005$.

niche adaptation (68), and could cause variability not only in the bacterial strains but also in the responding antibody repertoire. Hussell et al. $(69,70)$ showed that the extreme variability of $H$. pylori strains led to diverse $T$ cell responses. Moreover, they showed that B-cells did not respond to $H$. pylori themselves, but required contact-dependent help from $H$. pylori-specific $\mathrm{T}$ cells, and their Ig genes responded to auto-antigens, similar to our observations. Alternatively, the repertoires in the biopsies - even GHP biopsies may reflect immune responses to a variety of pathogens, including but not limited to $H$. pylori.

Although in many cases DLBCL is associated with MALT-L, the two types of lymphomas presented different dominant clone combinations and lineage tree characteristics. DLBCL may develop after prolonged stimulation during gastritis, derive from a lowgrade malignant clone, or it can initiate de novo, depending on the mutations in each clone. In this study, as MALT-L and DLBCL presented different dominant clone combinations, in contrast to our expectations; we speculate that in these cases DLBCL may have initiated de novo. MALT-L samples presented different lineage tree characteristics from those of all other conditions, although we expected MALT-L to resemble GHP. In fact, we identified preferential use of the autoreactive gene VH3-7 in MALT-L samples. VH3-7 was one of the common VH genes in GHP (but not the dominant clone). These findings suggest that gastric MALT-L is derived from highly restricted B-cell subsets probably resulting from specific antigenic stimulation, such as with $H$. pylori (15). It is possible that B-cells in MALT-L react with self-antigens (66), however, the role of self-antigens in the development of the malignancy has yet to be examined. Moreover, lineage tree drawings demonstrated longer trunks and path lengths in MALT-L dominant clones, compared with all other conditions. These differences in tree characteristics correlate with lower initial affinity and lower selection threshold, respectively. Low selection pressure may simply be the result of abundance of antigen, and this may indeed be the case in gastric MALT-L. The above may indicate that MALT-L has undergone a longer mutational history than other conditions. On the contrary, the shorter lengths observed in DLBCL dominant clones may be a result of shorter diversification and responses to specific (yet-unknown) antigens, with higher initial affinity compared to MALT-L and the two types of gastritis (21). The latter may be risk factors for aggressive lymphoma development.

We observed some similar $\mathrm{VH}-\mathrm{JH}$ combinations in all conditions, together with over-expressed and preferred combinations unique to gastritis, MALT-L, and DLBCL samples. These combinations should be investigated in order to further understand their role in the development of each condition. For example, the relatively extensive use of combinations, which were previously found in other malignancies, in DLBCL samples in this study reinforces the notion that some DLBCL clones had developed from MALT-L clones. Moreover, the fact that the dominant combinations were identical in all conditions except in MALT-L (and that MALT-L did not contain this combination at all) is interesting and should be further investigated. We also identified frequent $\mathrm{VH}$ genes in GHP repertoires and in the dominant clones in MALT-L samples, which were also found in autoimmune diseases. It was previously shown that some autoreactive $\mathrm{B}$ and $\mathrm{T}$ cells are activated during $H$. pylori infection (71). The connection between the appearances of these $\mathrm{VH}$ genes in GHP and MALT-L samples from our study and in autoimmune diseases remains to be explored. There was no prominent trend toward any V, D, or J gene family in the over-expressed combinations in each of the conditions. However, combinations that were over-expressed in lymphoma dominant clones compared to other conditions had a clear preference toward the use of $\mathrm{VH} 2$, JH1 in MALT-L and VH1, JH2 in DLBCL. The role of these combinations and gene families in lymphomas is still unknown.

It should be noted that, because we studied formalin-fixed paraffin-embedded archival biopsies, we had access to only a limited number of biopsies, and limited amounts of DNA, as DNA in the preserved biopsies is often denatured (72). More samples in more conditions will have to be studied in order to give a clearer picture of the roles of specific $\mathrm{V}(\mathrm{D}) \mathrm{J}$ genes and combinations in inflammation and malignancies. Moreover, the similarity between conditions is also affected by the limited number of samples in each condition. This may cause the similarity calculations to be biased toward random features of the samples that may characterize a certain condition, and thus affect the interpretations. However, the similarity between samples within each condition was not very large, so it is unlikely that some random feature is common to all samples of the same condition. Coincident with obtaining more samples, we intend to use the Illumina highthroughput sequencing (HTS) in future studies, in order to avoid the 454 sequencing artifacts, which resulted in discarding many sequences (73). A possible argument could be raised regarding the exclusion of duplicate sequences from our analysis. As mentioned in the Section "Materials and Methods," duplicate sequences may stem from the PCR amplification, and may cause misidentification of dominant clones. Although we stringently defined duplicate sequences as those that had the exact nucleotide composition 
and equal lengths, most of the sequences differed not only by their lengths, but also in the mutations they include. Table S5 in Supplementary Material summarizes the distributions of the number of sequences in the tree-nodes in the dominant and second dominant clones in lymphoma samples. As can be seen from Table S5 in Supplementary Material, most of the nodes contained only one sequence, implying that most of the sequences differed not only in their lengths, but also in the mutations they include. There were, however, several nodes that contained more than one sequence. This does not necessarily indicate that those sequences are duplicates, as the clone-tree is built according to the aligned sub-sequences that are shared between all sequences in the clone. This means that sequences included in a specific node could differ in their ends, and not necessarily be duplicates.

All conditions in this study presented similar mutation rates and the same SHM targeting motifs. Replacement and silent mutation analysis revealed a strong selection against replacement mutations in the CDR regions of all conditions. This may indicate that most of the examined IgH variable region gene sequences represented B-cell receptors (BCRs) that were already highly specific to their antigens and thus selection operated against replacement mutations in their CDR regions, which are responsible for antigen binding. These results are also consistent with previous work on lymphomas from our lab (30).

In this study, we used the Morisita similarity index in order to measure similarity between conditions (as mentioned in the Section "Materials and Methods"). Similarity measures were calculated between all clones in all samples in the compared conditions. In lymphomas, only the dominant clone(s) are relevant, as the rest of the clones in each sample represent other B-cells that are also present in the tissue, but are not related to the malignancy. Thus, the MALT-L and DLBCL conditions were not included in calculation of similarity measures exactly because the dominant clones in these conditions cannot be compared to the whole other samples in other conditions. In addition, Morisita similarity index is of order 2, which emphasizes large clones. This affects the similarity results. However, we are interested in the larger clones in each condition as they probably represent the dominant responses.

In summary, we showed that gastritis positive or negative for $H$. pylori presented very similar IgH variable region gene repertoires. This suggests that the diverse stomach repertories do not change much due to the presence of the bacteria, and moreover, GHP does not become oligoclonal (or at least with narrower repertoire) due to $H$. pylori. MALT-L, however, presented different and unique dominant clone gene combinations, which can result from specific antigenic stimulation. As was mentioned in the Section "Introduction," several studies showed that $H$. pylori causes gastritis, and suggested that prolonged gastritis can lead to MALT-L, and that prolonged MALT-L can develop into DLBCL. This flow and graduation of diseases led us to the assumption that the repertoires (VDJ combinations) in these conditions would be similar, because these conditions were initiated by the bacteria, and several clones got out of control to progress into lymphoma. Moreover, the diversity in these conditions was expected to be narrower than that in CLN, and to be progressively lower as the conditions proceed toward the aggressive lymphoma. However, the results differed from what we expected. In addition, some combinations did appear in several conditions, but not in MALT-L, and the DLBCL dominant clones also appear in other conditions (so they were not unique to the cancer). We speculate that the transformation into MALT-L, after the prolonged stimulation by the chronic GHP, amplified specific combination(s) that were also found in GHP but in a lower frequency (such as VH3-7). The two types of lymphomas differed in their dominant clone gene combinations and lineage tree characteristics, suggesting differences in the abundance of antigens, if not in their nature, which remain to be explored.

\section{MATERIALS AND METHODS \\ HISTOPATHOLOGICAL SPECIMENS}

Five gastric DLBCL biopsies, 3 gastric MALT-L biopsies, 10 chronic gastritis biopsies ( 3 with $H$. pylori background and 7 that were negative for $H$. pylori), and 19 reactive lymph node biopsies (which served as controls), each from a different patient, were selected from the pathology department archives in the Sheba Medical Center (Table S1 in Supplementary Material). Tissue biopsies were taken during resection procedures and were used in this study in accordance with institutional Helsinki committee guidelines and approval. Histochemical stains, by Hematoxylin-Eosin $(\mathrm{H} \& \mathrm{E})$ and Giemsa, were performed for histological evaluation and $H$. pylori identification. For diagnosis of lymphoproliferative disease and characterization of lymphocyte populations, immunohistochemical stains (e.g., CD20, CD3, CD23, CD21, cyclin-D1, $\mathrm{Ki67}$, and IgD) were also performed. All cases were revised by two independent pathologists to confirm the diagnosis.

\section{DNA EXTRACTION}

Paraffin-embedded blocks were cut using a microtome to get extremely thin slices of tissues (sections). Each of the biopsies was consecutively cut to yield 10-20 sections of $4 \mu$ each, depending on tissue size. All sections from each biopsy were inserted into an eppendorf tube with $200 \mu \mathrm{l}$ water (Sigma) and were heated in $90^{\circ} \mathrm{C}$ until the paraffin was melted. After the tubes were centrifuged at full speed (14000 rpm) for $1 \mathrm{~min}$, a paraffin ring was created and could easily be removed from each of the tubes. Water was drawn from the tubes while tissues remained in the tubes. Extraction of DNA was then performed using the QIAamp DNA Mini Kit (or the QIAamp DNA Micro Kit for very small samples) according to the QIAGEN protocol.

In some cases, a micro-dissection was needed. First, H\&E stained thin sections were reviewed by a pathologist, and areas of interest were outlined. The tissues were cut from 5 to 10 sections that were placed onto five slides per tissue. The slides were heated in $90^{\circ} \mathrm{C}$ for $15 \mathrm{~min}$. Next, the slides were hydrated $(5 \mathrm{~min}$ soaking in Xylene, brief immersion in Ethanol 100, 96, and 70\% in this order). The slides were then placed to dry. According to the outlined stained slides of each tissue, the hydrated slides were scratched with buffer ATL (QIAamp DNA Mini Kit, QIAGEN) and sample scrapings were picked up into eppendorf tubes. Extraction of DNA was then performed using the QIAamp DNA Mini Kit (or the QIAamp DNA Micro Kit for very small samples) according to the QIAGEN protocol.

\section{PCR AMPLIFICATION AND HIGH-THROUGHPUT SEQUENCING}

For each sample taken from each biopsy, semi-nested PCR was performed using the same forward primers for the two PCR rounds and two different reverse primers as described below. 
Forward primers from FR2 region:

VH1: 5'-TGCGMCAGGCCCCYGGACAAR-3', VH2: 5'-ARGRAAGGCCCTGGAGTGG-3', VH3: 5'-CCAGGCTCCAGGSAAG-3', VH4: 5'-MGGGAAGGGRCTGGAGTGG-3', VH5: 5'-GAAAGGCCTGGAGTGGATGGG-3', VH6: 5'-TTGAGTGGCTGGGRAGGAC-3'.

\section{Reverse primers:}

First round - JH1R: 5' -TGAGGAGACGGTGACCAGGGT-3', Second round - JH2R: 5'-TGACCRKGGTHCCYTGGCCC-3'.

There is no specific primer for VH7 gene family in the FR2 region, as the $\mathrm{VH} 7$ primer in this region is very similar to that of VH1, thus, it amplifies the VH1 family and creates a very strong VH1 bias.

The primers were augmented for HTS experiments by the addition of $5^{\prime}$ sequencing adapter elements and 10-nucleotide unique sample molecular identification (MID) tags according to the 454 FLX Titanium chemistry protocol (Roche) (74). Proofreading Taq DNA polymerase (ABgene) was used in PCR reactions according to the manufacturer's protocol. PCR reaction was performed on a sample of $50 \mathrm{ng}$ DNA from each sample, with slight changes according to calibration (because DNA was taken from different tissues, and each tissue can differ in the percentages of lymphocytes). PCR products were separated on a $2 \%$ agarose gel stained by ethidium bromide. Clear bands were cut from the gel and DNA was extracted using the MinElute Gel Extraction kit (QIAGEN), according to the manufacturer's protocol. Sequencing of small samples of the PCR products by the classic Sanger method (after cloning to pGEM - T-easy vector) was performed in order to make sure they are Ig gene amplifications and the sequences of the primers and the tags are intact. DNA concentration of PCR products from each sample were determined by PicoGreen dye and fluorospectrometer (Nanodrop). According to these results, a mixture containing $10^{9}$ molecules of PCR products from each sample was prepared and sent to sequencing. HTS was performed using the 454 GS FLX Titanium platform by DYN Diagnostics Ltd., the sole representative in Israel of Roche Diagnostics. Raw data files containing a total of $\sim 120,000$ reads were received when the HTS was completed. Raw data files can be downloaded from the SRA database, accession number PRJNA206548 (Runs: SRR873440, SRR873441, SRR873442).

\section{HTS RAW DATA PRE-PROCESSING}

To process the 454 raw data, we used our program Ig-HTScleaner (73). Ig-HTS-cleaner discards artifact sequences, assigns the sequences to samples according to their MID tags, identifies primers, and discards sequences much shorter or longer than the expected length of an Ig variable region gene, or sequences with average quality scores below a defined threshold. Parameters used in the Ig-HTS-Cleaner run were as follows. Average quality score threshold of 20, a maximum of 2 allowed mismatches in the primer search, $75 \%$ of the primer's length to search, and a range of 25 bases at the ends of the read for the MID and primers search (Table S3 in Supplementary Material).
Next, we discarded duplicate sequences, which are completely identical sequences, from each sample. We cannot exclude the possibility that duplicate sequences are a result of the PCR amplification; hence the existence of many identical sequences in a sample does not necessarily indicate that the sequence is found in the original biopsy in the same frequency.

Afterward, we used our program Ig-Indel-Identifier (Ig Insertion - Deletion Identifier) (73), in order to identify legitimate and artifact insertions and/or deletions (indels) in the sequences. Parameters used in the Ig-Indel-Identifier run were as follows. HPT length was set to 2, quality score threshold (for suspected point mutations) of 25 , and the number of sequences in the same clone containing the same indel was set to 1 (Table S4 in Supplementary Material). Table 4 presents the numbers of unique sequences from each condition after discarding sequences with suspected indels. These were the final numbers of sequences that were analyzed.

\section{GERMLINE VDJ SEGMENT IDENTIFICATION AND ASSIGNMENT INTO CLONES}

Clonally related sequences were identified by identical V(D)J segments and by highly homologous sequences of the CDR3 of their Ig genes. For gene segment identification, we used SoDA (75). We computationally grouped the sequences into clones based only on their $\mathrm{V}, \mathrm{D}$, and J segments. We aligned clonally related sequences using ClustalW2 (76), in order to confirm that the CDR3 in the clonally related sequences were highly homologous. If not, we separated the sequences into clonally related groups according to the different CDR3 sequences.

\section{REPERTOIRE ANALYSIS}

We enumerated the clones based on $\mathrm{V}(\mathrm{D}) \mathrm{J}$ combinations. Results are presented as the average sample percentages of clones of each $\mathrm{VH}-\mathrm{JH}$ combination, across all samples within the same group. Using the percentages normalizes for different numbers of sequences and/or clones, due to sampling of different numbers of B-cells or obtaining different DNA quantities in each case.

In order to examine the relationships between the VDJ combinations used in each repertoire, we needed to compare the observed repertoires to repertoires predicted under some model, for example, under the assumption that the expression of each gene in each VDJ combination (e.g., V1D1J1) is independent of that of other genes. Immunologists call this assumption "the product rule" (77). Deviations from this assumption can thus point at interdependencies between the $\mathrm{V}, \mathrm{D}$, and J genes. We decided to look only at the gene family level, as higher resolution (genes, alleles) would give extremely large numbers of possible combinations, far from the number of combinations observed and therefore the frequencies of each expected combination at the gene or allele level would be close to zero. Thus, each observed gene combination would be significantly different from the expected. Using only families of the VDJ segments would solve this problem. For each sample, we counted the number of unique sequences that used each V, D, or J family. We then calculated the frequency of each $V / D / J$ family as the number of unique sequences using this family divided by the total number of unique sequences in the sample. We then created all possible 
Table 4 | Number of unique sequences ${ }^{a}$, without suspected indels ${ }^{b}$, from each condition.

\begin{tabular}{|c|c|c|c|c|c|c|}
\hline & CLN & GNHP & GHP & MALT-L & DLBCL & Total \\
\hline Number of patients (samples) & 19 & 7 & 3 & 3 & 5 & 37 \\
\hline Number of unique sequences & 23,308 & 4,676 & 3,373 & 3,851 & 4,389 & 39,597 \\
\hline Range of sequences ${ }^{c}$ & $384-3,353$ & $75-1,105$ & $513-1,406$ & $838-1,854$ & $267-1,601$ & \\
\hline \multirow[t]{5}{*}{ Dominant clone sizes ${ }^{d}$} & & & & $360(249)$ & $162(49)$ & \\
\hline & & & & 461 (399) & $325(257)$ & \\
\hline & & & & $408(321)$ & $58(42)$ & \\
\hline & & & & & $78(47)$ & \\
\hline & & & & & 418 (193) & \\
\hline
\end{tabular}

a Unique sequence: a sequence that differs from all other sequences due to one or more insertion(s), deletion(s), or point mutation(s).

${ }^{b}$ After sequences with suspected indels were discarded.

'The lowest-to-highest numbers of unique sequences without suspected indels in each sample from each condition.

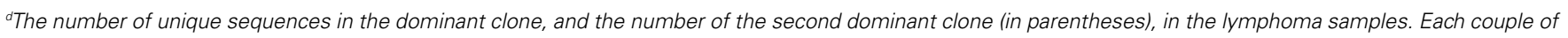
numbers represents one-sample.

combinations that can be made using the observed VDJ families, and defined their expected frequencies as the product of the $\mathrm{V} / \mathrm{D} / \mathrm{J}$ family frequencies calculated in the previous step. Next, we calculated the actual frequencies of the observed combinations (number of unique sequences in each observed combination divided by the total number of unique sequences). There was no point in creating combinations with families that did not appear in the sample, as there was no meaning of calculating frequencies of non-existing combinations.

In order to know whether a combination was expressed more or less than expected, we calculated the expression: $\log _{2}$ (observed/expected). If a specific combination was overexpressed compared to the expected frequency, the ratio inside the logarithm would be larger than 1 , as the observed frequency would be larger than the expected, and thus the logarithm would be positive. On the other hand, if a specific combination was underexpressed compared to the expected frequency, the ratio inside the logarithm would be smaller than 1, and thus the logarithm will be negative. Combinations that were not observed at all received the value $(-\infty)$, because the expression inside the logarithm was zero. This step was repeated for each sample. It is important to note that most of the combinations $(>80 \%)$ were observed in a significantly different frequency than expected. Out of these combinations, 99\% were under-represented (because the number of combinations observed is smaller than the potential number), and only $1 \%$ of the combinations were over-expressed compared to the expected frequency, and any such case of over-expression was thus particularly noticeable.

The final step was to unite all combinations from all samples as follows: we created a matrix, where rows represented samples and columns represented VDJ combinations. For each combination and for each sample, we inserted the logarithm that was calculated as above. If a sample did not have a specific combination, the cell would be left unfilled. The full matrix was used to carry out the statistical tests. In order to examine whether some combinations tend to appear more or less than expected, we carried out a one-sample $t$-test on each of the conditions. In order to examine differences in combination usage between conditions, we carried out a two-sample ANOVA test.
In order to graphically present repertoires, we only plotted $\mathrm{V}-\mathrm{J}$ repertoires, not showing the $\mathrm{DH}$ segments used in each $\mathrm{VH}-\mathrm{JH}$ combination. There are several ways of presenting also the $\mathrm{DH}$ genes used in the repertoires $(78,79)$. However, as mentioned above, DH segments are sometimes misidentified, so we preferred to focus on $\mathrm{V}$ and $\mathrm{J}$ segments.

\section{DIVERSITY ANALYSIS}

Clones in samples can be regarded as species in habitats

In the case of lymphocyte clonal repertoire samples (e.g., those obtained from tissue biopsies), we treat each sample as a sample from a habitat, in which the "species" are the BCR or TCR clones found in the sample. Each of the clones may be composed of a number of different sequences. In TCR clones, all sequences are identical, but in BCR clones sequences from the same clone may be different due to SHM, and one may choose to use only unique sequences found, or all sequences including multiplicate ones. The latter choice depends on whether identical sequences coming from different cells can be identified as such, or cannot be distinguished from sequence duplications caused by PCR amplification. If the former is true (as when using random barcoding in the PCR primers), then the number of sequences that come from different cells is known, and can be used to estimate diversity. If not, then TCR diversity cannot be estimated, and BCR diversity can only be estimated based on the numbers of unique sequences and thus would usually only gives a minimum estimate of the total diversity, as we have done in this study.

\section{Diversity indices}

In order to quantify the diversity of clonal repertoires (such as antibody/BCR or TCR gene repertoires) in each experimental or clinical condition, and later to be able to compare between two or more conditions, we used diversity indices (such as the Species Richness, the Shannon entropy, or the Simpson concentration, which are indices of order 0,1 , and 2, respectively) (80). These indices take into account the number of species and (in indices of order $>0$ ) the frequency of members of a species (in our case, sequences) of each species (in our case, clone) in each habitat sample. In indices of order 0 , diversity is defined simply as number of 
species (in our case, lymphocyte clones) in a sample. In order 1 indices, clone size (or frequency) is taken into account, as in the Shannon entropy when diversity is the sum of $\left[-p_{\mathrm{i}}^{*} \ln \left(p_{\mathrm{i}}\right)\right]$, where $i$ represents a species or clone and $p_{i}$ represents its size (the number of members/sequences, see below). Order 2 indices attribute more weight to large clones, as in the Simpson concentration, which is the sum of $p_{\mathrm{i}}^{2}$. In our studies, we used both order 1 and 2 diversity indices, i.e., the Shannon entropy and the Simpson concentration.

\section{Diversity measures}

From the sample diversity indices, we have calculated the alpha, beta, and gamma diversity measures for each condition (80). The alpha diversity measure represents the average sample diversity in each condition/population. In order to calculate alpha, we calculate the alpha diversity of each sample, and then average over all samples from the same condition. The gamma diversity measure represents the "global" repertoire diversity across all samples studied in each condition/population. It is calculated as the diversity of the pool containing all the sequences from all the samples from the same condition/population.

Finally, the beta diversity measure, which represents the diversity component resulting from the variability between samples, is derived from the alpha and gamma measures using the method of Jost et al. (80). The beta diversity measure is calculated as the gamma diversity of each condition/population divided by the alpha diversity (average of the diversities of individual samples). In order to allow an intuitive comparison between the diversities of each of the groups, all the diversity measures can be expressed as their number equivalents (80), which reflect the number of equally sized clones needed to produce the given value of the diversity index.

\section{Estimating the full repertoire from the sample}

Considering the large number of sequences that were observed only once in each sample, it is likely that many rare clones in an individual's original full repertoire were not detected. To account for the presence of unobserved "species" (clones), all diversity measures can be estimated for whole repertoires (rather than calculated for the sample) using the method described by Chao and Shen (81), which is based on a non-parametric estimation of diversity indices where there are undetected species. Chao and Shen's approach utilizes the concept of sample coverage to adjust the diversity indices for clones that escaped sampling. The sample coverage is estimated from the proportion of species/sequences that are observed only once within a sample.

In our Ig gene repertoire studies, the abundance data (numbers of unique sequences) of antibody clones in each sample were used to estimate the mean, standard error, and 95\% CI of the total number of unique sequences in clones within each sample. This was done using SPADE®, a program designed for diversity calculations (81). The alpha diversity for each sample, and the gamma diversities for combined samples in each condition, were then calculated from the order 1 or 2 diversity indices of the estimated total repertoires, also using SPADE@(81). In principle, beta is calculated as the average alpha of all samples in the condition divided by the gamma of the condition, as explained above. In order to compare between conditions, however, we needed to calculate CI for beta. This was done by calculating beta index per sample (alpha of the sample divided by the gamma of the condition) and then calculating the CI for each condition (Figure 5).

\section{SIMILARITY ANALYSIS}

Another method we used to compare between conditions is the Morisita similarity index (82). SPADE@(81) was used to calculate a similarity matrix, in which we measured each individual repertoire's similarity to all other individual repertoires. The average of similarity indices of individuals in a given group to those in another group represents the similarity index for the comparison between the two groups. A value close to 1 represents high similarity between two groups, and a value close to 0 represents low similarity.

The highest values of the Morisita similarity indices representing the highest similarity were rather low and relatively far from 1 , indicating the sensitivity of this method. However, they were consistent with observed repertoire diversities.

\section{Ig LINEAGE TREE ANALYSES}

Clonally related Ig gene sequences from each sample were used to create mutational lineage trees using our program IgTree@(83), as described in previous work $(29,30)$. All trees were measured using our program MTree $\odot$, quantifying the graphical properties of the trees $(84,85)$. A thorough statistical analysis has concluded that seven specific tree characteristics possess the highest correlation values with the biological parameters and are hence most informative (67). As described there, these properties are the minimum root to leaf path length, the average distance from a leaf to the first split node/fork, the average outgoing degree, that is the average number of branches coming out of any node, the root's outgoing

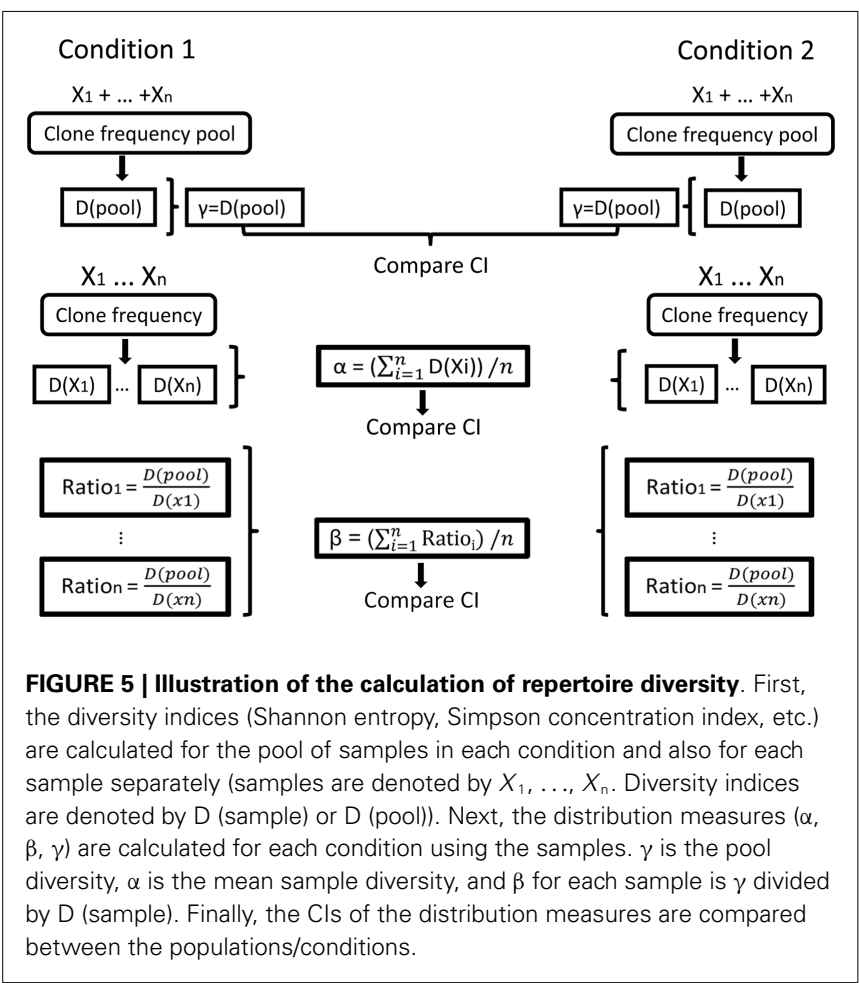


degree, minimum distance between adjacent split nodes/forks, the length of the tree's trunk and minimum distance from the root to any split node/fork. The analysis in this study has thus focused on these properties. Comparison between lineage tree characteristics of different conditions was done using the non-parametric Mann-Whitney $U$-test, as normal distributions (required by tests such as Student's $t$-test) could not be assumed. We used the FDR correction (86) for multiple comparisons.

Replacement (R) and silent (S) mutation analysis methods attempt to measure the extent of selection operating on the diversifying clones. These methods compare the frequencies of replacement mutations found in the frame-work and CDR regions of mutated Ig gene sequences to their expected frequency, based on codon usage of the germline sequence. We used the updated focused binomial test by Hershberg et al. $(87,88)$. The numbers of observed mutations were pooled for each data group by IgTree $\odot$, and the new focused binomial formula (88) was calculated using Microsoft Excel@. This measure was also performed for each sample separately, yielding the same results; however, when comparing conditions, we chose to show the pooled analysis for simplicity. Additional mutational analyses were carried out as described in previous studies $(27,28,30)$, however, no significant differences between the conditions were found.

\section{AUTHOR CONTRIBUTIONS}

Miri Michaeli performed all steps from DNA extraction from samples to bioinformatical analysis of the sequences, and wrote the manuscript. Hilla Tabibian-Keissar supervised the molecular process. Ginette Schiby revised the samples to confirm the diagnosis. Gitit Shahaf and Yishai Pickman developed the diversity analysis. Lena Hazanov developed the bioinformatical analyses. Kinneret Rosenblatt was in charge of the laboratory in which the molecular work was performed. Deborah K. Dunn-Walters advised the author throughout the study. Ramit Mehr and Iris Barshack supervised the molecular work and the analyses performed, and finalized the manuscript. All authors read and approved the final manuscript.

\section{ACKNOWLEDGMENTS}

This work was supported in parts by an Israel Science Foundation (grant number 270/09, to Ramit Mehr); and a Human Frontiers Science Program Research Grant (to Ramit Mehr and Deborah K. Dunn-Walters). The work was part of Miri Michaeli's studies toward a combined M.Sc/Ph.D. degree in Bar-Ilan University, and she was supported by a Combined Technologies M.Sc Scholarship from the Israeli Council for Higher Education.

\section{SUPPLEMENTARY MATERIAL}

The Supplementary Material for this article can be found online at http://www.frontiersin.org/Journal/10.3389/fimmu.2014.00264/ abstract

\section{REFERENCES}

1. Schubert TT, Schubert AB, MA CK. Symptoms, gastritis, and Helicobacter pylori in patients referred for endoscopy. Gastrointest Endosc (1992) 38:357-60. doi:10.1016/S0016-5107(92)70432-5

2. Kusters JG, van Vliet AHM, Kuipers EJ. Pathogenesis of Helicobacter pylori infection. Clin Microbiol Rev (2006) 19:449-90. doi:10.1128/CMR.00054-05
3. Sipponen P, Hyuarinen H. Role of Helicobacter pylori in the pathogenesis of gastritis, peptic ulcer and gastric cancer. Scand J Gastroenterol (1993) 28:3-6. doi:10.3109/00365529309098333

4. Veldhuyzen van Zanten SJ, Sherman PM. Helicobacter pylori infection as a cause of gastritis, duodenal ulcer, gastric cancer and nonulcer dyspepsia: a systematic overview. Can Med Assoc J (1994) 150:177-85.

5. Nordenstedt H, Graham DY, Kramer JR, Rugge M, Verstovsek G, Fitzgerald S, et al. Helicobacter pylori-negative gastritis: prevalence and risk factors. Am J Gastroenterol (2013) 108:65-71. doi:10.1038/ajg.2012.372

6. Ryan JL, Shen Y-J, Morgan DR, Thorne LB, Kenney SC, Dominguez RL, et al. Epstein-Barr virus infection is common in inflamed gastrointestinal mucosa. Dig Dis Sci (2012) 57:1887-98. doi:10.1007/s10620-012-2116-5

7. Karlsson FA, Burman P, Loof L, Mardh S. Major parietal cell antigen in autoimmune gastritis with pernicious anemia is the acid-producing $\mathrm{H}+, \mathrm{K}+-$ adenosine triphosphatase of the stomach. J Clin Invest (1988) 81:475-9. doi:10.1172/JCI113344

8. Sipponen P, Kosunen TU, Valle J, Riihelä M, Seppälä K. Helicobacter pylori infection and chronic gastritis in gastric cancer. J Clin Pathol (1992) 45:319-23. doi:10.1136/jcp.45.4.319

9. Segal ED, Cha J, Lo J, Falkow S, Tompkins LS. Altered states: involvement of phosphorylated CagA in the induction of host cellular growth changes by Helicobacter pylori. Proc Natl Acad Sci U S A (1999) 96:14559-64. doi:10.1073/pnas. 96.25.14559

10. Lin W-C, Tsai H-F, Kuo S-H, Wu M-S, Lin C-W, Hsu P-I, et al. Translocation of Helicobacter pylori CagA into human B lymphocytes, the origin of mucosa-associated lymphoid tissue lymphoma. Cancer Res (2010) 70:5740-8. doi:10.1158/0008-5472.CAN-09-4690

11. Fujimori K, Shimodaira S, Akamatsu T, Furihata K, Katsuyama T, Hosaka S. Effect of Helicobacter pylori eradication on ongoing mutation of immunoglobulin genes in gastric MALT lymphoma. Br J Cancer (2005) 92:312-9. doi:10. 1038/sj.bjc.6602262

12. Suzuki H, Saito Y, Hibi T. Helicobacter pylori and gastric mucosa-associated lymphoid tissue (MALT) lymphoma: updated review of clinical outcomes and the molecular pathogenesis. Gut Liver (2009) 3:81-7. doi:10.5009/gnl.2009.3.2.81

13. Lochhead P, El-Omar E. Helicobacter pylori infection and gastric cancer. Best Pract Res Clin Gastroenterol (2007) 21:281-97. doi:10.1016/j.bpg.2007.02.002

14. Wotherspoon AC, Ortiz Hidalgo C, Falzon MR, Isaacson PG. Helicobacter pylori-associated gastritis and primary B-cell gastric lymphoma. Lancet (1991) 338:1175-6. doi:10.1016/0140-6736(91)92035-Z

15. Zucca E, Bertoni F, Roggero E, Bosshard G, Cazzaniga G, Pedrinis E, et al. Molecular analysis of the progression from Helicobacter pylori-associated chronic gastritis to mucosa-associated lymphoid-tissue lymphoma of the stomach. N Engl J Med (1998) 338:804-10. doi:10.1056/NEJM199803193381205

16. Isaacson PG. Lymphomas of mucosa associated lymphoid tissue (MALT). Am J Surg Pathol (1992) 16:201-5. doi:10.1097/00000478-199202000-00023

17. Cavalli F, Isaacson PG, Gascoyne RD, Zucca E. MALT lymphomas. Hematology Am Soc Hematol Educ Program (2001) 2001:241-58. doi:10.1182/asheducation2001.1.241

18. Chan JKC, Ng CS, Isaacson PG. Relationship between high-grade lymphoma and low-grade B-cell mucosa-associated lymphoid tissue lymphoma (MALToma) of the stomach. Am J Pathol (1990) 136:1153-64.

19. Peng H, Du M, Diss TC, Isaacson PG, Pan L. Genetic evidence for a clonal link between low and high-grade components in gastric MALT B-cell lymphoma. Histopathology (1997) 30:425-9. doi:10.1046/j.1365-2559.1997.5450786.x

20. Freeman C, Berg JW, Cutler SJ. Occurrence and prognosis of extranodal lymphomas. Cancer (1972) 29:252-60. doi:10.1002/1097-0142(197201)29:1<252: :AID-CNCR2820290138>3.0.CO;2- \\#

21. Go JH, Kim DS, Kim TJ, Ko YH, Ra HK, Rhee JC, et al. Comparative studies of somatic and ongoing mutations in immunoglobulin heavy-chain variable region genes in diffuse large B-cell lymphomas of the stomach and the small intestine. Arch Pathol Lab Med (2003) 127:1443-50. doi:10.1043/1543-2165(2003) 127<1443:CSOSAO > 2.0.CO;2

22. Alizadeh AA, Eisen MB, Davis RE, Ma C, Lossos IS, Rosenwald A, et al. Distinct types of diffuse large B-cell lymphoma identified by gene expression profiling. Nature (2000) 403:503-11. doi:10.1038/35000501

23. De Paepe P, De Wolf-Peeters C. Diffuse large B-cell lymphoma: a heterogeneous group of non-Hodgkin lymphomas comprising several distinct clinicopathological entities. Leukemia (2007) 21:37-43. doi:10.1038/sj.leu.2404449 
24. Banerjee M, Mehr R, Belelovsky A, Spencer J, Dunn-Walters DK. Age- and tissue-specific differences in human germinal center B cell selection revealed by analysis of IgVH gene hypermutation and lineage trees. Eur J Immunol (2002) 32:1947-57. doi:10.1002/1521-4141(200207)32:7<1947::AID-IMMU1947>3. $0 . \mathrm{CO} ; 2-1$

25. Steiman-Shimony A, Edelman H, Barak M, Shahaf G, Dunn-Walters DK, Stott DI, et al. Immunoglobulin variable-region gene mutational lineage tree analysis: application to autoimmune diseases. Autoimmun Rev (2006) 5:242-51. doi:10.1016/j.autrev.2005.07.008

26. Steiman-Shimony A, Edelman H, Hutzler A, Barak M, Zuckerman NS, Shahaf G, et al. Lineage tree analysis of immunoglobulin variable-region gene mutations in autoimmune diseases: chronic activation, normal selection. Cell Immunol (2006) 244:130-6. doi:10.1016/j.cellimm.2007.01.009

27. Zuckerman NS, Hazanov H, Barak M, Edelman H, Hess S, Shcolnik H, et al. Somatic hypermutation and antigen-driven selection of B cells are altered in autoimmune diseases. J Autoimmun (2010) 35:325-35. doi:10.1016/j.jaut.2010. 07.004

28. Zuckerman NS, Howard WA, Bismuth J, Gibson KL, Edelman H, Berrih-Aknin S, et al. Ectopic GC in the thymus of myasthenia gravis patients show characteristics of normal GC. Eur Immunol (2010) 40:1150-61. doi:10.1002/eji.200939914

29. Tabibian-Keissar H, Zuckerman NS, Barak M, Dunn-Walters DK, SteimanShimony A, Chowers Y, et al. B-cell clonal diversification and gut-lymph node trafficking in ulcerative colitis revealed using lineage tree analysis. Eur J Immunol (2008) 38:2600-9. doi:10.1002/eji.200838333

30. Zuckerman NS, McCann KJ, Ottensmeier CH, Barak M, Shahaf G, Edelman $\mathrm{H}$, et al. Ig gene diversification and selection in follicular lymphoma, diffuse large B cell lymphoma and primary central nervous system lymphoma revealed by lineage tree and mutation analyses. Int Immunol (2010) 22:875-87. doi:10.1093/intimm/dxq441

31. Manske MK, Zuckerman NS, Timm MM, Maiden S, Edelman H, Shahaf G, et al. Quantitative analysis of clonal bone marrow CD19+ B cells: use of B cell lineage trees to delineate their role in the pathogenesis of light chain amyloidosis. Clin Immunol (2006) 120:106-20. doi:10.1016/j.clim.2006.01.008

32. Abraham RS, Manske MK, Zuckerman NS, Sohni A, Edelman H, Shahaf G, et al. Novel analysis of clonal diversification in blood B cell and bone marrow plasma cell clones in immunoglobulin light chain amyloidosis. J Clin Immunol (2007) 27:69-87. doi:10.1007/s10875-006-9056-9

33. Gurrieri C, McGuire P, Zan H, Yan X-J, Cerutti A, Albesiano E, et al. Chronic lymphocytic leukemia B cells can undergo somatic hypermutation and intraclonal immunoglobulin $\mathrm{V}(\mathrm{H}) \mathrm{DJ}(\mathrm{H})$ gene diversification. J Exp Med (2002) 196:629-39. doi:10.1084/jem.20011693

34. Kostareli E, Sutton L, Hadzidimitriou A, Darzentas N, Kouvatsi A, Tsaftaris A, et al. Intraclonal diversification of immunoglobulin light chains in a subset of chronic lymphocytic leukemia alludes to antigen-driven clonal evolution. Leukemia (2010) 24:1317-24. doi:10.1038/leu.2010.90

35. Bashford-Rogers RJM, Palser AL, Huntly BJ, Rance R, Vassiliou GS, Follows GA, et al. Network properties derived from deep sequencing of human B-cell receptor repertoires delineate B-cell populations. Genome Res (2013) 23(11):1874-84. doi:10.1101/gr.154815.113

36. Zhu D, Orchard J, Oscier DG, Wright DH, Stevenson FK. V(H) gene analysis of splenic marginal zone lymphomas reveals diversity in mutational status and initiation of somatic mutation in vivo. Blood (2002) 100:2659-61. doi:10.1182/blood-2002-01-0169

37. Traverse-Glehen A, Davi F, Ben Simon E, Callet-Bauchu E, Felman P, Baseggio L, et al. Analysis of $\mathrm{VH}$ genes in marginal zone lymphoma reveals marked heterogeneity between splenic and nodal tumors and suggests the existence of clonal selection. Haematologica (2005) 90:470-8.

38. Matolcsy A, Schattner EJ, Knowles DM, Casali P. Clonal evolution of B cells in transformation from low- to high-grade lymphoma. Eur J Immunol (1999) 29:1253-64. doi:10.1002/(SICI)1521-4141(199904)29:04<1253::AIDIMMU1253>3.0.CO;2-8

39. Carlotti E, Wrench D, Matthews J, Iqbal S, Davies A, Norton A, et al. Transformation of follicular lymphoma to diffuse large B-cell lymphoma may occur by divergent evolution from a common progenitor cell or by direct evolution from the follicular lymphoma clone. Blood (2009) 113:3553-7. doi:10.1182/blood2008-08-174839

40. Green MR, Gentles AJ, Nair RV, Irish JM, Kihira S, Liu CL, et al. Hierarchy in somatic mutations arising during genomic evolution and progression of follicular lymphoma. Blood (2013) 121:1604-11. doi:10.1182/blood-2012-09457283

41. Wündisch T, Thiede C, Alpen B, Stolte M, Neubauer A. Are lymphocytic monoclonality and immunoglobulin heavy chain $(\mathrm{IgH})$ rearrangement premalignant conditions in chronic gastritis? Microsc Res Tech (2001) 53:414-8. doi:10.1002/jemt.1110

42. Miyamoto M, Haruma K, Hiyama T, Kamada T, Masuda H, Shimamoto F, et al. High incidence of B-cell monoclonality in follicular gastritis: a possible association between follicular gastritis and MALT lymphoma. Virchows Arch (2002) 440:376-80. doi:10.1007/s00428-001-0575-8

43. Georgopoulos SD, Triantafyllou K, Fameli M, Kitsanta P, Spiliadi C, Anagnostou D, et al. Molecular analysis of B-cell clonality in Helicobacter pylori gastritis. Dig Dis Sci (2005) 50:1616-20. doi:10.1007/s10620-005-2905-1

44. Bahler DW, Szankasi P, Kulkarni S, Tubbs RR, Cook JR, Swerdlow SH. Use of similar immunoglobulin VH gene segments by MALT lymphomas of the ocular adnexa. Mod Pathol (2009) 22:833-8. doi:10.1038/modpathol.2009.42

45. Walsh SH, Rosenquist R. Immunoglobulin gene analysis of mature B-cell malignancies: reconsideration of cellular origin and potential antigen involvement in pathogenesis. Med Oncol (2005) 22:327-41. doi:10.1385/MO:22:4:327

46. Fais F, Ghiotto F, Hashimoto S, Sellars B, Valetto A, Allen SL, et al. Chronic lymphocytic leukemia B cells express restricted sets of mutated and unmutated antigen receptors. J Clin Invest (1998) 102:1515-25. doi:10.1172/ JCI3009

47. Yamashita Y, Kajiura D, Tang L, Hasegawa Y, Kinoshita T, Nakamura S, et al. $\mathrm{XCR} 1$ expression and biased $\mathrm{VH}$ gene usage are distinct features of diffuse large B-cell lymphoma initially manifesting in the bone marrow. Am J Clin Pathol (2011) 135:556-64. doi:10.1309/AJCPCTDC5PY3LXBP

48. Bende RJ, Aarts WM, Riedl RG, de Jong D, Pals ST, van Noesel CJM. Among B cell non-Hodgkin's lymphomas, MALT lymphomas express a unique antibody repertoire with frequent rheumatoid factor reactivity. J Exp Med (2005) 201:1229-41. doi:10.1084/jem.20050068

49. Pimentel BJ, Stefanoff CG, Moreira AS, Seuánez HN, Zalcberg IR. Use of V H, $\mathrm{D}$ and $\mathrm{J} \mathrm{H}$ immunoglobulin gene segments in Brazilian patients with chronic lymphocytic leukaemia (CLL). Genet Mol Biol (2008) 31:643-8. doi:10.1590/ S1415-47572008000400007

50. Matsuda F, Shin EK, Nagaoka H, Matsumura R, Haino M, Fukita Y, et al. Structure and physical map of 64 variable segments in the 3'0.8-megabase region of the human immunoglobulin heavy-chain locus. Nat Genet (1993) 3:88-94. doi:10.1038/ng0193-88

51. Johnson TA, Rassenti LZ, Kipps TJ. Ig VH1 genes expressed in B cell chronic lymphocytic leukemia exhibit distinctive molecular features. J Immunol (1997) 158:235-46.

52. Bayerl MG, Bentley G, Bellan C, Leoncini L, Ehmann WC, Palutke M. Lacunar and Reed-Sternberg-like cells in follicular lymphomas are clonally related to the centrocytic and centroblastic cells as demonstrated by laser capture microdissection. Am J Clin Pathol (2004) 122:858-64. doi:10.1309/PMR86PHKK4J3RUH3

53. Hashimoto Y, Tsukamoto N, Nakahashi H, Yokohama A, Saitoh T, Handa H, et al. Hairy cell leukemia-related disorders consistently show low CD27 expression. Pathol Oncol Res (2009) 15:615-21. doi:10.1007/s12253-009-9161-1

54. Nakamura-Kikuoka S, Takahi K, Tsuboi H, Toyosaki-Maeda T, Maeda-Tanimura $\mathrm{M}$, Wakasa $\mathrm{C}$, et al. Limited VH gene usage in B-cell clones established with nurse-like cells from patients with rheumatoid arthritis. Rheumatology (2006) 45:549-57. doi:10.1093/rheumatology/kei170

55. Bahler DW, Swerdlow SH. Clonal salivary gland infiltrates associated with myoepithelial sialadenitis (Sjögren's syndrome) begin as nonmalignant antigenselected expansions. Blood (1998) 91:1864-72.

56. Brezinschek HP, Foster SJ, Brezinschek RI, Dörner T, Domiati-Saad R, Lipsky PE. Analysis of the human VH gene repertoire. Differential effects of selection and somatic hypermutation on human peripheral CD5(+)/IgM+ and CD5(-)/IgM+ B cells. J Clin Invest (1997) 99:2488-501. doi:10.1172/JCI119433

57. Wu Y-C, Kipling D, Leong HS, Martin V, Ademokun A, Dunn-Walters DK. High-throughput immunoglobulin repertoire analysis distinguishes between human IgM memory and switched memory B-cell populations. Blood (2010) 116:1070-8. doi:10.1182/blood-2010-03-275859

58. Perotti M, Ghidoli N, Altara R, Diotti RA, Clementi N, De Marco D, et al. Hepatitis $\mathrm{C}$ virus (HCV)-driven stimulation of subfamily-restricted natural IgM antibodies in mixed cryoglobulinemia. Autoimmun Rev (2008) 7:468-72. doi:10.1016/j.autrev.2008.03.008 
59. Mao Z, Quintanilla-Martinez L, Raffeld M, Richter M, Krugmann J, Burek $\mathrm{C}$, et al. IgVH mutational status and clonality analysis of Richter's transformation: diffuse large B-cell lymphoma and Hodgkin lymphoma in association with B-cell chronic lymphocytic leukemia (B-CLL) represent 2 different pathways of disease evolution. Am J Surg Pathol (2007) 31:1605-14. doi:10.1097/PAS.0b013e31804bdaf8

60. Sakuma H, Nakamura T, Uemura N, Chiba T, Sugiyama T, Asaka M, et al. Immunoglobulin $\mathrm{VH}$ gene analysis in gastric MALT lymphomas. Mod Pathol (2007) 20:460-6. doi:10.1038/modpathol.3800758

61. Lenze D, Greiner A, Knörr C, Anagnostopoulos I, Stein H, Hummel M. Receptor revision of immunoglobulin heavy chain genes in human MALT lymphomas. Mol Pathol (2003) 56:249-55. doi:10.1136/mp.56.5.249

62. Alpen B, Wündisch T, Dierlamm J, Börsch G, Stolte M, Neubauer A. Clonal relationship in multifocal non-Hodgkin's lymphoma of mucosa-associated lymphoid tissue (MALT). Ann Hematol (2004) 83:124-6. doi:10.1007/s00277-0030763-5

63. Siakantaris MP, Pangalis GA, Dimitriadou E, Kontopidou FN, Vassilakopoulos TP, Kalpadakis C, et al. Early-stage gastric MALT lymphoma: is it a truly localized disease? Oncologist (2009) 14:148-54. doi:10.1634/theoncologist.2008-0178

64. De Wolf-Peeters C, Achten R. The histogenesis of large-cell gastric lymphomas. Histopathology (1999) 34:71-5. doi:10.1046/j.1365-2559.1999.00602.x

65. Friedberg JW. Diffuse large B-cell lymphoma. J Hematol Oncol (2008) 22:941-52. doi:10.1016/j.hoc.2008.07.002

66. Lenze D, Berg E, Volkmer-Engert R, Weiser A, Greiner A, Knörr-Wittmann $\mathrm{C}$, et al. Influence of antigen on the development of MALT lymphoma. Blood (2006) 107:1141-8. doi:10.1182/blood-2005-04-1722

67. Shahaf G, Barak M, Zuckerman NS, Swerdlin N, Gorfine M, Mehr R. Antigendriven selection in germinal centers as reflected by the shape characteristics of immunoglobulin gene lineage trees: a large-scale simulation study. J Theor Biol (2008) 255:210-22. doi:10.1016/j.jtbi.2008.08.005

68. Salau L, Linz B, Suerbaum S, Saunders NJ, Saunders N. The diversity within an expanded and redefined repertoire of phase-variable genes in Helicobacter pylori. Microbiology (2004) 150:817-30. doi:10.1099/mic.0.26993-0

69. Hussell T, Isaacson PG, Crabtree JE, Spencer J. The response of cells from lowgrade B-cell gastric lymphomas of mucosa-associated lymphoid tissue to Helicobacter pylori. Lancet (1993) 342:571-4. doi:10.1016/0140-6736(93)91408-E

70. Hussell T, Isaacson PG, Crabtree JE, Spencer J. Helicobacter pylori-specific tumour-infiltrating $\mathrm{T}$ cells provide contact dependent help for the growth of malignant B cells in low-grade gastric lymphoma of mucosa-associated lymphoid tissue. J Pathol (1996) 178:122-7. doi:10.1002/(SICI)1096-9896(199602) 178:2<122::AID-PATH486>3.0.CO;2-D

71. Ernst PB, Gold BD. The disease spectrum of Helicobacter pylori: the immunopathogenesis of gastroduodenal ulcer and gastric cancer. Annu Rev Microbiol (2000) 54:615-40. doi:10.1146/annurev.micro.54.1.615

72. Tabibian-Keissar H, Schibby G, Michaeli M, Rakovsky-Shapira A, AzoguiRosenthal N, Dunn-Walters DK, et al. PCR amplification and high throughput sequencing of immunoglobulin heavy chain genes from formalin-fixed paraffinembedded human biopsies. Exp Mol Pathol (2012) 94:182-7. doi:10.1016/j. yexmp.2012.08.002

73. Michaeli M, Noga H, Tabibian-Keissar H, Barshack I, Mehr R. Automated cleaning and pre-processing of immunoglobulin gene sequences from highthroughput sequencing. Front Immunol (2012) 3:386. doi:10.3389/fimmu.2012. 00386

74. Ansorge WJ. Next-generation DNA sequencing techniques. N Biotechnol (2009) 25:195-203. doi:10.1016/j.nbt.2008.12.009

75. Volpe JM, Cowell LG, Kepler TB. SoDA: implementation of a 3D alignment algorithm for inference of antigen receptor recombinations. Bioinformatics (2006) 22:438-44. doi:10.1093/bioinformatics/btk004
76. Larkin MA, Blackshields G, Brown NP, Chenna R, McGettigan PA, McWilliam $\mathrm{H}$, et al. Clustal W and Clustal X version 2.0. Bioinformatics (2007) 23:2947-8. doi:10.1093/bioinformatics/btm404

77. Mehr R, Sternberg-Simon M, Michaeli M, Pickman Y. Models and methods for analysis of lymphocyte repertoire generation, development, selection and evolution. Immunol Lett (2012) 148:11-22. doi:10.1016/j.imlet.2012.08.002

78. Weinstein JA, Jiang N, White RA, Fisher DS, Quake SR. High-throughput sequencing of the zebrafish antibody repertoire. Science (2009) 324:807-10. doi:10.1126/science. 1170020

79. Briney BS, Willis JR, McKinney BA, Crowe JE. High-throughput antibody sequencing reveals genetic evidence of global regulation of the naïve and memory repertoires that extends across individuals. Genes Immun (2012) 13:469-73. doi:10.1038/gene.2012.20

80. Jost L. Partitioning diversity into independent alpha and beta components. Ecology (2007) 88:2427-39. doi:10.1890/06-1736.1

81. Chao A, Shen TJ. Program SPADE (Species Prediction and Diversity Estimation): Program and User's Guide. (2003). Available from: http://chao.stat.nthu.edu.tw

82. Chao A, Jost L, Chiang SC, Jiang Y-H, Chazdon RL. A two-stage probabilistic approach to multiple-community similarity indices. Biometrics (2008) 64:1178-86. doi:10.1111/j.1541-0420.2008.01010.x

83. Barak M, Zuckerman NS, Edelman H, Unger R, Mehr R. IgTree: creating immunoglobulin variable region gene lineage trees. J Immunol Methods (2008) 338:67-74. doi:10.1016/j.jim.2008.06.006

84. Dunn-Walters DK, Belelovsky A, Edelman H, Banerjee M, Mehr R. The dynamics of germinal centre selection as measured by graph-theoretical analysis of mutational lineage trees. Dev Immunol (2002) 9:233-43. doi:10.1080/ 10446670310001593541

85. Dunn-Walters DK, Edelman H, Mehr R. Immune system learning and memory quantified by graphical analysis of B-lymphocyte phylogenetic trees. Biosystems (2004) 76:141-55. doi:10.1016/j.biosystems.2004.05.011

86. Benjamini Y, Hochberg Y. Controlling the false discovery rate: a practical and powerful approach to multiple testing. J R Stat Soc Ser B (1995) 57:289-300.

87. Hershberg U, Uduman M, Shlomchik MJ, Kleinstein SH. Improved methods for detecting selection by mutation analysis of $\mathrm{Ig} \mathrm{V}$ region sequences. Int Immunol (2008) 20:683-94. doi:10.1093/intimm/dxn026

88. Uduman M, Yaari G, Hershberg U, Stern JA, Shlomchik MJ, Kleinstein SH. Detecting selection in immunoglobulin sequences. Nucleic Acids Res (2011) 39:W499-504. doi:10.1093/nar/gkr413

Conflict of Interest Statement: The authors declare that the research was conducted in the absence of any commercial or financial relationships that could be construed as a potential conflict of interest.

Received: 10 December 2013; paper pending published: 17 January 2014; accepted: 20 May 2014; published online: 03 June 2014.

Citation: Michaeli M, Tabibian-Keissar H, Schiby G, Shahaf G, Pickman Y, Hazanov L, Rosenblatt K, Dunn-Walters DK, Barshack I and Mehr R (2014) Immunoglobulin gene repertoire diversification and selection in the stomach - from gastritis to gastric lymphomas. Front. Immunol. 5:264. doi: 10.3389/fimmu.2014.00264

This article was submitted to B Cell Biology, a section of the journal Frontiers in Immunology.

Copyright (C) 2014 Michaeli, Tabibian-Keissar, Schiby, Shahaf, Pickman, Hazanov, Rosenblatt, Dunn-Walters, Barshack and Mehr. This is an open-access article distributed under the terms of the Creative Commons Attribution License (CC BY). The use, distribution or reproduction in other forums is permitted, provided the original author(s) or licensor are credited and that the original publication in this journal is cited, in accordance with accepted academic practice. No use, distribution or reproduction is permitted which does not comply with these terms. 